\title{
A LORENTZIAN SURFACE IN A FOUR-DIMENSIONAL MANIFOLD OF NEUTRAL SIGNATURE AND ITS REFLECTOR LIFT
}

\author{
KAZUYUKI HASEGAWA
}

Communicated by Vasil V. Tsanov

\begin{abstract}
A Lorentzian surface in a four-dimensional manifold of neutral signature is called super-extremal if its reflector lift is horizontal. We give an elementary proof of a rigidity theorem for super-extremal surfaces in the space of constant curvature and neutral signature. As corollary, a characterization of the immersion of the Veronese type is given.
\end{abstract}

\section{Introduction}

The twistor lifts play an important role for oriented surfaces in oriented fourdimensional Riemannian manifolds and have been studied by many researchers (see [1,3-5, 7-10] for example). In geometry of pseudo-Riemannian manifold of neutral signature, the reflector bundle is the corresponding object to the twistor space. For Lorentzian surfaces in four-dimensional manifolds of neutral signature, the reflector lifts are defined in [12], which are corresponding to the twistor lifts in Riemannian case. In this paper, we study Lorentzian surfaces in four-dimensional manifolds of neutral signature with horizontal reflector lifts, which are corresponding to superminimal surfaces in Riemannian geometry. In pseudo-Riemannian geometry, because of the failure of definiteness for metrics, different situations often occur from Riemannian cases. For example, in Riemannian case, a connected minimal surface of constant Gaussian curvature in the Euclidean space must be flat and an open part of a two-plane (see [2]). But one can find many non-totally geodesic extremal flat surfaces in the pseudo-Euclidean space of neutral signature (see Section 4), where extremal means vanishing of the mean curvature vector field. We say that a Lorentzian surface is super-extremal if its reflector lift is horizontal. Note that the notions of the reflector lift and super-extremal surface can be defined for higher even-dimentional cases. In [11], a rigidity theorem for super-extremal surfaces is obtained.

The purpose of this paper is to give several examples of surfaces such that their reflector lifts are horizontal or para-holomorphic and an elementary proof of a 
rigidity theorem in a low dimensional case, that is, in the case of super-extremal surfaces in the four-dimensional space forms $Q_{2}^{4}(c)$ of constant curvature $c$ and neutral signature.

\section{Preliminaries}

Throughout this paper, all manifolds and maps are assumed to be smooth. Let $E$ be a vector bundle over a manifold $M$ and $E_{x}$ the fiber of $E$ over $x \in M$. We write $T P$ for the tangent bundle of a manifold $P$. For vector bundles $E, E^{\prime}$ over $M$, we denote the homomorphism bundle whose fiber is the space of linear mappings $E_{x}$ to $E_{x}^{\prime}$ by $\operatorname{Hom}\left(E, E^{\prime}\right)$, and set $\operatorname{End}(E):=\operatorname{Hom}(E, E)$. The space of all sections of a vector bundle $E$ is denoted by $\Gamma(E)$. Let $\varphi: N \rightarrow M$ be a smooth map and $E$ a vector bundle over $M$. The pull back bundle of $E$ by $\varphi$ is denoted by $\varphi^{\#} E$.

In this section, we recall some definitions and equations for pseudo-Riemannian manifolds and submanifolds. Let $\tilde{M}$ be a pseudo-Riemannian manifold with a fixed pseudo-Riemannian metric $\tilde{g}$. A tangent vector $X$ of $\tilde{M}$ is called spacelike if $\tilde{g}(X, X)>0$ or $X=0$, null if $\tilde{g}(X, X)=0$ and $X \neq 0$, and timelike if $\tilde{g}(X, X)<0$. The set of all null vectors at $x \in \tilde{M}$ is called the nullcone at $x \in \tilde{M}$, which is denoted by $\Lambda_{\tilde{g}}(x)$. If $\operatorname{dim} \tilde{M}-\nu=\nu$, then we say that $\tilde{M}$ is of neutral signature, where $\nu$ is the index of $\tilde{g}$. We call a psudo-Riemannian manifold $\tilde{M}$ Lorentzian if $\nu=1$.

Let $(M, g)$ be a pseudo-Riemannian submanifold in $(\tilde{M}, \tilde{g})$. We denote the LeviCivita connection of $\tilde{g}$ (respectively $g$ ) by $\tilde{\nabla}$ (respectively $\nabla$ ). Let $\nabla^{\perp}$ be the normal connection of the normal bundle $T^{\perp} M$. Let $\alpha$ and $A$ be the second fundamental form and the shape operator of $M$. The mean curvature vector field of $M$ is denoted by $H$. We define $\nabla^{\prime} \alpha$ by the equality

$$
\left(\nabla_{X}^{\prime} \alpha\right)(Y, Z)=\nabla_{X}^{\perp} \alpha(Y, Z)-\alpha\left(\nabla_{X} Y, Z\right)-\alpha\left(Y, \nabla_{X} Z\right)
$$

for all $X, Y, Z \in \Gamma(T M)$. Let $\tilde{R}, R$ and $R^{\perp}$ be the curvatures forms of $\tilde{\nabla}, \nabla$ and $\nabla^{\perp}$, respectively. Then the following equations hold

$$
\begin{aligned}
\tilde{g}(\tilde{R}(X, Y) Z, W)= & g(R(X, Y) Z, W)+g^{\perp}(\alpha(X, Z), \alpha(Y, W)) \\
& -g^{\perp}(\alpha(X, W), \alpha(Y, Z)) \\
\tilde{g}(\tilde{R}(X, Y) Z, \xi)= & g^{\perp}\left(\left(\nabla_{X}^{\prime} \alpha\right)(Y, Z), \xi\right)-g^{\perp}\left(\left(\nabla_{Y}^{\prime} \alpha\right)(X, Z), \xi\right) \\
\tilde{g}(\tilde{R}(X, Y) \xi, \zeta)= & g^{\perp}\left(R^{\perp}(X, Y) \xi, \zeta\right)+g\left(A_{\xi} X, A_{\zeta} Y\right)-g\left(A_{\xi} Y, A_{\zeta} X\right)
\end{aligned}
$$

for all $X, Y \in T M$ and $\xi, \zeta \in T^{\perp} M$, where $g^{\perp}$ is the metric of the normal bundle $T^{\perp} M$. We say that $M$ is totally geodesic (respectively totally umbilic) if $\alpha=0$ 
(respectively $\alpha(X, Y)=g(X, Y) H$ for all $X, Y \in T M)$. If $H=0$, then $M$ is called an extremal submanifold ( [17]). Note that, in [12], a submanifold with $H=0$ is called string. Let $\mathbb{R}_{\nu}^{n}$ be the pseudo-Euclidean space of the dimension $n$ and the index $\nu$ with the flat standard metric. Let $\left(x^{1}, \ldots, x^{n+1}\right)$ be the standard coordinate on $\mathbb{R}^{n+1}$. The pseudosphere $S_{\nu}^{n}(r)$ of the index $\nu$ and the radius $r>0$ is defined by

$$
S_{\nu}^{n}(r)=\left\{p \in \mathbb{R}_{\nu}^{n+1} ;-\sum_{i=1}^{\nu}\left(x^{i}(p)\right)^{2}+\sum_{j=\nu+1}^{n+1}\left(x^{j}(p)\right)^{2}=r^{2}\right\} .
$$

Similarly, the pseudohyperbolic space $H_{\nu}^{n}(r)$ of the index $\nu$ and the radius $r>0$ is defined by

$$
H_{\nu}^{n}(r)=\left\{p \in \mathbb{R}_{\nu+1}^{n+1} ;-\sum_{i=1}^{\nu+1}\left(x^{i}(p)\right)^{2}+\sum_{j=\nu+2}^{n+1}\left(x^{j}(p)\right)^{2}=-r^{2}\right\} .
$$

The all spaces $\mathbb{R}_{\nu}^{n}, \mathrm{~S}_{\nu}^{n}(r)$ and $H_{\nu}^{n}(r)$ are of constant curvature $0,1 / r^{2},-1 / r^{2}$. We denote the space form by $Q_{\nu}^{n}(c)$ which is one of $\mathbb{R}_{\nu}^{n}, \mathrm{~S}_{\nu}^{n}(r)$ or $H_{\nu}^{n}(r)$, where $n$ is the dimension, $\nu$ is the index and $c$ is constant curvature of $Q_{\nu}^{n}(c)$.

\section{Reflector Bundles and Reflector Lifts}

Let $(\tilde{M}, \tilde{g})$ be an oriented four-dimensional manifold of neutral signature. The Hodge star operator is denoted by $*$. Since $*^{2}=$ id on the space of two-forms $\Lambda^{2}(\tilde{M})$, we have

$$
\Lambda^{2}(\tilde{M})=\Lambda_{+}^{2}(\tilde{M}) \oplus \Lambda_{-}^{2}(\tilde{M})
$$

where $\Lambda_{ \pm}^{2}(\tilde{M})=\left\{\omega \in \Lambda^{2}(\tilde{M}) ; * \omega= \pm \omega\right\}$. Let $\left(e_{1}, \ldots, e_{4}\right)$ of $\tilde{M}$ be an orthonormal frame which is compatible with the orientation and $\tilde{g}\left(e_{i}, e_{i}\right)=\varepsilon_{i}$, $\varepsilon_{1}=\varepsilon_{2}=-1, \varepsilon_{3}=\varepsilon_{4}=1$. We denote its dual frame by $\left(\omega^{1}, \ldots, \omega^{4}\right)$. Set $s_{1}:=\omega^{1} \wedge \omega^{2}-\omega^{3} \wedge \omega^{4}, s_{2}:=\omega^{1} \wedge \omega^{3}-\omega^{2} \wedge \omega^{4}, s_{3}:=\omega^{1} \wedge \omega^{4}+\omega^{2} \wedge \omega^{3}$. Then $s_{1}, s_{2}, s_{3}$ is an orthonormal frame of $\Lambda_{-}^{2}(\tilde{M})$. Let $J_{i} \in \Gamma(\operatorname{End}(T \tilde{M}))$ be the endomorphism corresponding to $s_{i}(i=1,2,3)$. Then we have $J_{1}\left(e_{1}\right)=$ $-e_{2}, J_{1}\left(e_{3}\right)=-e_{4}$ and so on. It is easy to see that $\left(J_{1}\right)^{2}=-I,\left(J_{2}\right)^{2}=I$, $\left(J_{3}\right)^{2}=I$ and $J_{3}=J_{2} J_{1}=-J_{1} J_{2}$. Let $Q$ be the vector subbundle of $\operatorname{End}(T \tilde{M})$ which is locally spanned by $J_{1}, J_{2}, J_{3}$. We have $\tilde{g}\left(J_{1}, J_{1}\right)=1, \tilde{g}\left(J_{2}, J_{2}\right)=-1$, $\tilde{g}\left(J_{3}, J_{3}\right)=-1$, that is, the fiber metric of $Q$ has the index two. It it easy to see that $Q$ is a parallel subbundle in $\operatorname{End}(T \tilde{M})$ with respect to the connection which is induced by the Levi-Civita connection $\tilde{\nabla}$ of $\tilde{M}$. We use the same letter $\tilde{\nabla}$ for the connection of $\operatorname{End}(T \tilde{M})$ induced by $\tilde{\nabla}$. 
Lemma 1. Set $J=a J_{1}(x)+b J_{2}(x)+c J_{3}(x)$ at each $x \in \tilde{M}(a, b, c \in \mathbb{R})$. The following statements are mutually equivalent: 1) $\left.J^{2}=I, 2\right)-a^{2}+b^{2}+c^{2}=1$, 3) $\tilde{g}_{x}(J, J)=-1$.

We define the reflector bundle $Z$ by

$$
Z=\bigcup_{x \in M}\left\{J \in Q_{x} ; \tilde{g}_{x}(J, J)=-1\right\} .
$$

The bundle projection $p: Z \rightarrow \tilde{M}$ and the Levi-Civita connection $\tilde{\nabla}$ on $\tilde{M}$ induce the decomposition $T Z=T^{h} Z \oplus T^{v} Z$ into the horizontal subbundle $T^{h} Z$ and the vertical subbundle $T^{v} Z$. On the reflector bundle $Z$, the almost para-complex (or bilagrangian) structure $J^{Z}$ is defined by $J^{Z}(X)=\left(J\left(p_{*}(X)\right)\right)_{J}^{h}$ for all horizontal vector $X$ at $J \in Z$ and $J^{Z}(V)=J^{v}(V)$ for all vertical vector $V$, where $Y^{h}$ is the horizontal lift of $Y \in T M$ and $J^{v}$ is the canonical para-complex structure on each fiber $\simeq H_{1}^{2}(1)$.

Let $f:(M, g) \rightarrow(\tilde{M}, \tilde{g})$ be an isometric immersion from an oriented two dimensional Lorentzian manifold $(M, g)$ into an oriented four-dimensional manifold $(\tilde{M}, \tilde{g})$ of neutral signature. Using an orthonormal frame $e_{1}, e_{2}, e_{3}, e_{4}$ adapted to the orientation of $\tilde{M}$ such that $e_{1}, e_{3}$ defines the orientation of $M$ and $e_{2}, e_{4}$ are normal to $M$, we define $J: T M \rightarrow T M$ by $J\left(e_{1}\right)=-e_{3}$ and $J\left(e_{3}\right)=-e_{1}$, and $J^{\perp}: T^{\perp} M \rightarrow T^{\perp} M$ by $J^{\perp}\left(e_{2}\right)=e_{4}$ and $J^{\perp}\left(e_{4}\right)=e_{2}$. Such frame $e_{1}, e_{2}, e_{3}, e_{4}$ is said to be adapted. We define $\tilde{J} \in \Gamma\left(f^{\#} Q\right)$ by

$$
\tilde{J}(X):=J(X) \text { and } \tilde{J}(\zeta):=J^{\perp}(\zeta)
$$

for $X \in T M$ and $\zeta \in T^{\perp} M$. Then $\tilde{J} \in \Gamma\left(f^{\#} Q\right)$ is called the reflector lift of $M$ (see [12]). Hereafter, we often omit the symbol " $f$ " for the induced objects of the immersion $f$ if there is no confusion for the simplicity. For reflector bundles and reflector lifts, see [12].

Lemma 2. The para-complex structures $J$ and $J^{\perp}$ are parallel with respect to $\nabla$ and $\nabla^{\perp}$ respectively.

Here we define surfaces corresponding to superminimal and twistor holomorphic surfaces in Riemannian geometry. A surface $M$ in $\tilde{M}$ is called super-extremal if its reflector lift is horizontal, that is, $\tilde{\nabla} \tilde{J}=0$, where $\tilde{\nabla}$ is the induced connection on $f^{\#} Q$ from the Levi-Civita connection of $\tilde{M}$. By the similar way to the Riemannian case and using Lemma 2, we have

Lemma 3. A surface $M$ is super-extremal if and only if the second fundamental form $\alpha$ satisfies $\alpha(X, J Y)-J^{\perp} \alpha(X, Y)=0$ for all $X, Y \in T M$. 
A surface $M$ in $\tilde{M}$ is called isotropic with negative spin if its reflector lift is paraholomorphic (or bilagrangian), that is, $\tilde{J}_{*} \circ J=J^{Z} \circ \tilde{J}_{*}$ (precisely, $\left(f_{\#} \circ \tilde{J}\right)_{*} \circ J=$ $\left.J^{Z} \circ\left(f_{\#} \circ \tilde{J}\right)_{*}\right)$, see [12]. A surface is called isotropic with positive spin if the reflector lift is para-holomorphic with respect to the opposite orientation. We also obtain

Lemma 4. A surface $M$ is isotropic with negative spin if and only if the second fundamental form $\alpha$ satisfies

$$
J^{\perp} \alpha(J X, J Y)-\alpha(J X, Y)-\alpha(X, J Y)+J^{\perp} \alpha(X, Y)=0, \quad X, Y \in T M .
$$

After proving fundamental lemmas for super-extremal and isotropic surfaces with negative spin, we give examples of such surfaces in the next section. We define $\beta$ and $\gamma$ by

$$
\beta(X, Y)=\alpha(X, J Y)-J^{\perp} \alpha(X, Y)
$$

and

$$
\gamma(X, Y)=J^{\perp} \alpha(J X, J Y)-\alpha(J X, Y)-\alpha(X, J Y)+J^{\perp} \alpha(X, Y)
$$

for $X, Y \in T M$. Let $K$ be the Gaussian curvature of $M$. We define the normal curvature function $K^{\perp}$ by $K^{\perp}=g^{\perp}\left(R^{\perp}\left(e_{1}, e_{3}\right) e_{4}, e_{2}\right)$ and a function $\rho$ by

$$
\rho=g^{\perp}\left(\alpha\left(e_{1}, e_{3}\right)+J^{\perp} \alpha\left(e_{1}, e_{1}\right), \alpha\left(e_{1}, e_{3}\right)+J^{\perp} \alpha\left(e_{3}, e_{3}\right)\right)
$$

where $\left(e_{1}, \ldots, e_{4}\right)$ is an adapted frame. Note that $\rho=0$ if $M$ is super-extremal. We summarize the fundamental formulae which we use in this paper. These are obtained by the straightforward calculations. By the definition of $\rho$ and (3), we have

$$
\rho=-\operatorname{det} A_{e_{2}}+\operatorname{det} A_{e_{4}}+K^{\perp}-\tilde{g}\left(\tilde{R}\left(e_{1}, e_{3}\right) e_{4}, e_{2}\right) .
$$

By (1), it is easy to see

$$
K=-\operatorname{det} A_{e_{2}}+\operatorname{det} A_{e_{4}}-\tilde{g}\left(\tilde{R}\left(e_{1}, e_{3}\right) e_{3}, e_{1}\right) .
$$

Combined with these equations, we have

$$
\rho-K=K^{\perp}-\tilde{g}\left(\tilde{R}\left(e_{1}, e_{3}\right) e_{4}, e_{2}\right)+\tilde{g}\left(\tilde{R}\left(e_{1}, e_{3}\right) e_{3}, e_{1}\right)
$$




\section{Examples and a Rigidity Theorem for Super-Extremal Surfaces}

Let $(M, g)$ be an oriented Lorentzian surface in an oriented four-dimensional pseudo-Riemannian manifold $(\tilde{M}, \tilde{g})$ of neutral signature, which is isometrically immersed by $f$. Let $J \in \Gamma(\operatorname{End}(T M))\left(\right.$ respectively $J^{\perp} \in \Gamma\left(\operatorname{End}\left(T^{\perp} M\right)\right)$ ) be the para-complex structure on $M$ (respectively $\left.T^{\perp} M\right)$. We set $T_{\varepsilon}^{\top}:=\operatorname{Ker}(J-\varepsilon I)$ and $T_{\varepsilon}^{\perp}:=\operatorname{Ker}\left(J^{\perp}-\varepsilon I\right)(\varepsilon= \pm 1)$. Let $p_{\varepsilon}^{\top}$ (respectively $p_{\varepsilon}^{\perp}$ ) be the projection from $T M$ (respectively $\left.T^{\perp} M\right)$ onto $T_{\varepsilon}^{\top}$ (respectively $T_{\varepsilon}^{\perp}$ ) $(\varepsilon= \pm 1$ ). The projections $p_{\varepsilon}^{\top}$ (respectively $p_{\varepsilon}^{\perp}$ ) are given by $p_{\varepsilon}^{\top}=(1 / 2)(I+\varepsilon J)$ (respectively $\left.p_{\varepsilon}^{\perp}=(1 / 2)\left(I+\varepsilon J^{\perp}\right)\right)(\varepsilon= \pm 1)$. It is easy to prove the following lemma.

Lemma 5. We have

1) $T_{\varepsilon}^{\top}$ and $T_{\varepsilon}^{\perp}$ are the parallel subbundles of $T M$ and $T^{\perp} M$ respectively $(\varepsilon=$ $\pm 1)$.

2) $T M=T_{1}^{\top} \oplus T_{-1}^{\top}$ and $T^{\perp} M=T_{1}^{\perp} \oplus T_{-1}^{\perp}$

3) $\Lambda_{g} \cup\{0\}=T_{1}^{\top} \cup T_{-1}^{\top}$ and $\Lambda_{g^{\perp}} \cup\{0\}=T_{1}^{\perp} \cup T_{-1}^{\perp}$.

By Lemma 5, we obtain

Lemma 6. For $X \in T_{1}^{\top}$ and $Y \in T_{-1}^{\top}$, we have

$$
\alpha(X, Y)=g(X, Y) H .
$$

By the straightforward calculations, we have the following lemmas.

Lemma 7. If $X \in T_{1}^{\top}$ and $Y \in T_{-1}^{\top}$, then we have

$$
\begin{aligned}
\beta(X, X) & =\alpha(X, X)-J^{\perp} \alpha(X, X) \\
\beta(Y, Y) & =-\alpha(Y, Y)-J^{\perp} \alpha(Y, Y) \\
\beta(X, Y) & =-g(X, Y)\left(H+J^{\perp} H\right) \\
\beta(Y, X) & =g(X, Y)\left(H-J^{\perp} H\right) .
\end{aligned}
$$

Lemma 8. If $X \in T_{1}^{\top}$ and $Y \in T_{-1}^{\top}$, then we have

$$
\begin{aligned}
\gamma(X, X) & =2\left(J^{\perp} \alpha(X, X)-\alpha(X, X)\right) \\
\gamma(Y, Y) & =2\left(J^{\perp} \alpha(Y, Y)+\alpha(Y, Y)\right) \\
\gamma(X, Y) & =0 .
\end{aligned}
$$


The following fact is proved in [12] using a local frame.

Lemma 9. A surface $M$ is super-extremal if and only if $M$ is extremal and isotropic with negative spin.

Proof: Assume that $M$ is super-extremal. Then we have $\alpha(X, X) \in T_{1}^{\perp}$ for all $X \in T_{1}^{\top}$ and $\alpha(Y, Y) \in T_{-1}^{\perp}$ for all $Y \in T_{-1}^{\top}$ by Lemma 3, (2) and (3). Then $M$ is isotropic with negative spin. Moreover, it follows that $H \in T_{1}^{\perp} \cap T_{-1}^{\perp}$ from (4) and (5). Hence, by Lemma 5, we have $H=0$. Next, we assume that $M$ is extremal and isotropic with negative spin. By Lemmas 7 and 8 , we see $\beta=0$, that is, $M$ is super-extremal.

By Lemma 8, we have

Proposition 1. A surface $M$ is isotropic with negative spin if and only if it holds that $\alpha(X, X) \in T_{1}^{\perp}$ for all $X \in T_{1}^{\top}$ and $\alpha(Y, Y) \in T_{-1}^{\perp}$ for all $Y \in T_{-1}^{\top}$.

Hence, to check if the surface is isotropic with a negative spin, it is sufficient to consider the second fundamental form with respect to null directions. Using Proposition 1, we see that the following immersions is isotropic with negative spin.

Example 1. Let $U$ be an open set of $\mathbb{R}_{1}^{2}$. We consider the immersion $f: U \rightarrow \mathbb{R}_{2}^{4}$ by

$f(x, y)=(a(x)+c(y) b(x), b(x)-c(y) a(x), a(x)-c(y) b(x), b(x)+c(y) a(x))$

where $a, b, c$ are functions defined on open intervals with $c^{\prime}\left(a^{\prime} b-a b^{\prime}\right) \neq 0$. Since

$$
\begin{aligned}
& f_{*}\left(\partial_{x}\right)=\left(a^{\prime}+c b^{\prime}, b^{\prime}-c a^{\prime}, a^{\prime}-c b^{\prime}, b^{\prime}+c a^{\prime}\right) \\
& f_{*}\left(\partial_{y}\right)=\left(c^{\prime} b,-c^{\prime} a,-c^{\prime} b, c^{\prime} a\right)
\end{aligned}
$$

and we have also $\tilde{g}\left(f_{*}\left(\partial_{x}\right), f_{*}\left(\partial_{x}\right)\right)=0, \tilde{g}\left(f_{*}\left(\partial_{y}\right), f_{*}\left(\partial_{y}\right)\right)=0, \tilde{g}\left(f_{*}\left(\partial_{x}\right), f_{*}\left(\partial_{y}\right)\right)$ $=-2 c^{\prime}\left(a^{\prime} b-a b^{\prime}\right)$. Moreover, we obtain

$$
\begin{aligned}
& \tilde{\nabla}_{\partial_{x}} \partial_{x}=\left(a^{\prime \prime}, b^{\prime \prime}, a^{\prime \prime}, b^{\prime \prime}\right)+c\left(b^{\prime \prime},-a^{\prime \prime},-b^{\prime \prime}, a^{\prime \prime}\right) \\
& \tilde{\nabla}_{\partial_{x}} \partial_{y}=c^{\prime}\left(b^{\prime},-a^{\prime},-b^{\prime}, a^{\prime}\right) \\
& \tilde{\nabla}_{\partial_{y}} \partial_{y}=c^{\prime \prime}(b,-a,-b, a) .
\end{aligned}
$$

Besides, it holds that

$$
\tilde{g}\left(\alpha\left(\partial_{x}, \partial_{x}\right) \alpha\left(\partial_{x}, \partial_{x}\right)\right)=0, \quad \alpha\left(\partial_{x}, \partial_{y}\right)=c^{\prime}\left(b^{\prime},-a^{\prime},-b^{\prime}, a^{\prime}\right), \quad \alpha\left(\partial_{y}, \partial_{y}\right)=0
$$


Therefore, by Proposition 1, we see that $f$ is an isotropic immersion with negative spin. By Lemma 6, we have

$$
H=-\frac{1}{2\left(a^{\prime} b-a b^{\prime}\right)}\left(b^{\prime},-a^{\prime},-b^{\prime}, a^{\prime}\right) .
$$

Hence the mean curvature vector field is null.

We see that $f$ is totally umbilic if and only if, for any null geodesic $c$ on $M$, the curve $f \circ c$ is geodesic. In fact, if $\alpha(X, X)=0$ for all $X \in T_{1}^{\top}$ and $\alpha(Y, Y)=0$ for all $Y \in T_{-1}^{\top}$, then it holds that $\alpha(Z, W)=g(Z, W) H$ for all $Z, W \in T M$ by Lemma 6. Consider

$$
U_{2}^{4}\left(x_{0}\right):=\left\{x \in \mathbb{R}_{2}^{4} ; \tilde{g}(x, x)=0, \tilde{g}\left(x, x_{0}\right)=-1\right\}
$$

for a null vector $x_{0} \in \mathbb{R}_{2}^{4}$. Then $U_{2}^{4}\left(x_{0}\right)$ is a flat totally umbilic surface in $\mathbb{R}_{2}^{4}$ with parallel null mean curvature vector field (see [13]). In Example 1, when $a(x)=$ $1, b(x)=x, c(y)=y$, the immersion is totally umbilic. In fact, this immersion is locally congruent to $U_{2}^{4}\left(x_{0}\right)$. In terms of null geodesics on $M$, we have the following corollary.

Corollary 1. Let $M$ be an oriented Lorentzian surface in an oriented four-dimensional pseudo-Riemannian manifold $\tilde{M}$ of neutral signature. Then the following statements are mutually equivalent:

1) $M$ is isotropic with negative spin.

2) For any $\varepsilon$ and any null geodesic con $M$ with $c^{\prime} \in T_{\varepsilon}^{\top}$, the curve $f \circ c$ satisfies $(f \circ c)^{\prime \prime}(t) \in\left(T_{\varepsilon}^{\perp}\right)_{c(t)}$ for all $t \in \operatorname{Dom}(c)$.

Next we give examples of super-extremal surfaces.

Example 2. If $(\tilde{M}, \tilde{g})$ is a four-dimensional para-Kähler manifold with the parallel para-complex structure $J^{\prime} \in \Gamma(Q)$. If $f_{*} \circ J=J^{\prime} \circ f_{*}$, then $M$ is a super-extremal surface. For example, $f: U \rightarrow \mathbb{R}_{2}^{4}$ defined by

$$
f(x, y)=(a(x)+c(y), b(x)+d(y), a(x)-c(y), b(x)-d(y))
$$

is super-extremal, where $U$ is an open set in $\mathbb{R}_{1}^{2}$ and $a, b, c, d$ are functions defined on open intervals such that $a^{\prime} c^{\prime}+b^{\prime} d^{\prime} \neq 0$.

Example 3. We define an extremal immersion $f: S_{1}^{2}(1) \rightarrow S_{2}^{4}(1 / \sqrt{3})$ by

$$
f(x, y, z)=\left(x y, z x, y z, \frac{\sqrt{3}}{6}\left(2 x^{2}+y^{2}+z^{2}\right), \frac{1}{2}\left(y^{2}-z^{2}\right)\right)
$$


which is corresponding to the Veronese immerison in Riemannian geometry (see [14]) where more general situations are considered. See also [15]. Note that the Veronese immerison in Riemannian geometry is a typical example of superminimal immersions. Next using Corollary 1 , we show that $f$ is isotropic with negative spin. It is sufficient to consider null geodesics passing through one point $p=(0,1,0)$. Null geodesics passing through $p$ can be written by $\gamma_{ \pm}(t)=(t, 1, \pm t)$ for $t \in \mathbb{R}$. Then we have

$$
\left(f \circ \gamma_{ \pm}\right)(t)=\left(t, \pm t^{2}, \pm t, \frac{\sqrt{3}}{6}\left(3 t^{2}+1\right), \frac{1}{2}\left(1-t^{2}\right)\right)
$$

for $t \in \mathbb{R}$. Therefore we have $\left(f \circ \gamma_{ \pm}\right)^{\prime \prime}(t)=(0, \pm 2,0, \sqrt{3},-1)$, and hence, $\left(f \circ \gamma_{ \pm}\right)^{\prime \prime}(t)$ are null vector and $\tilde{g}\left(\left(f \circ \gamma_{-}\right)^{\prime \prime}(0),\left(f \circ \gamma_{+}\right)^{\prime \prime}(0)\right) \neq 0$. Then $f$ is isotropic with negative spin and extremal, that is, $f$ is super-extremal by Lemma 9. Composing homotheties and anti-isometries of $\mathrm{S}_{1}^{2}(1)$ and $\mathrm{S}_{2}^{4}(1 / \sqrt{3})$, we can obtain super-extremal immersions of the Veronese type from $Q_{1}^{2}(c)$ to $Q_{2}^{4}(3 c)(c \neq 0)$.

The notions of the reflector lift and super-extremal surface can be defined for higher even-dimentional cases. In [11], a rigidity theorem for super-extremal surfaces is obtained in such cases. For the low dimensional case, we give more elementary proof of the rigidity theorem. To do this, we prepare a lemma for connections of a pseudo-Riemannian vector bundle. Let $E$ be a pseudo-Riemannian vector bundle with fiber metric $g^{E}$ over a pseudo-Riemannian manifold with the LeviCivita connection $\nabla$, and $\nabla^{\prime}$ metric connections of $E$. Let $\alpha$ be an $E$-valued symmetric tensor and its covariant derivative $\nabla^{\prime} \alpha$ induced by $\nabla^{\prime}$ and $\nabla$. We define $d^{\nabla^{\prime}} \alpha$ by

$$
\left(d^{\nabla^{\prime}} \alpha\right)(X, Y, Z):=\left(\nabla_{X}^{\prime} \alpha\right)(Y, Z)-\left(\nabla_{Y}^{\prime} \alpha\right)(X, Z)
$$

for $X, Y, Z \in \Gamma(T M)$. We note that $\alpha$ is $d^{\nabla^{\prime}}$-closed, that is, $d^{\nabla^{\prime}} \alpha=0$ if and only if the connection satisfies the equation of the Codazzi type. The following lemma can be proved in a similar way as Theorem 1 in [16].

Lemma 10. Let $E$ be a pseudo-Riemannian vector bundle with fiber metric $g^{E}$ over a pseudo-Riemannian manifold with the Levi-Civita connection $\nabla$, and $\nabla^{1}$, $\nabla^{2}$ metric connections of E. Let $\alpha$ be an E-valued symmetric tensor which satisfies $d^{\nabla^{1}} \alpha=0$ and $d^{\nabla^{2}} \alpha=0$. If $E_{x}=\operatorname{Span}\left\{\alpha(X, Y) ; X, Y \in T_{x} M\right\}$ for all $x \in M$, then $\nabla^{1}=\nabla^{2}$.

Here we can prove the following theorem.

Theorem 2. Let $f, \bar{f}: M \rightarrow Q_{2}^{4}(c)$ be super-extremal immersions from a Lorentzian surface $M$ such that both normal curvatures do not vanish at any point of $M$. Then there exist an isometry $\Phi$ of $Q_{2}^{4}(c)$ such that $\bar{f}=\Phi \circ f$. 
Proof: The corresponding objects associated with $\bar{f}$ are denoted by the symbol with "-", for example, $\overline{T^{\perp} M}$ is the normal bundle of $\bar{f}$. By (4), we have $K+$ $K^{\perp}=c$, so $c \neq K$. On the other hand, for nonzero vectors $X \in T_{1}^{\top}$ and $Y \in$ $T_{-1}^{\top}$, we see that $g^{\perp}(\alpha(X, X), \alpha(Y, Y)) \neq 0$. The first normal spaces coincide with the normal spaces, that is, $T^{\perp} M=\operatorname{Span}\{\alpha(X, X), \alpha(Y, Y)\}$ and $\overline{T^{\perp} M}=$ $\operatorname{Span}\{\bar{\alpha}(X, X), \bar{\alpha}(Y, Y)\}$. We define an isomorphism $\varphi: T^{\perp} M \rightarrow \overline{T^{\perp} M}$ by $\varphi(\alpha(X, X))=\bar{\alpha}(X, X)$ and $\varphi(\alpha(Y, Y))=\bar{\alpha}(Y, Y)$. Then we have

$$
g^{\perp}(\alpha(X, X), \alpha(X, X))=\bar{g}^{\perp}(\bar{\alpha}(X, X), \bar{\alpha}(X, X))
$$

and

$$
g^{\perp}(\alpha(Y, Y), \alpha(Y, Y))=\bar{g}^{\perp}(\bar{\alpha}(Y, Y), \bar{\alpha}(Y, Y)) .
$$

We see that $\varphi$ preserves the metrics of normal bundles. In fact, using by the Gauss equation (1), we have

$$
c g(X, Y)^{2}=K g(X, Y)^{2}+g^{\perp}(\alpha(X, X), \alpha(Y, Y))
$$

and hence, we have $g^{\perp}(\alpha(X, X), \alpha(Y, Y))=\bar{g}^{\perp}(\bar{\alpha}(X, X), \bar{\alpha}(Y, Y))(\neq 0)$. Therefore we see that $\varphi$ is isometry. Since both $f$ and $\bar{f}$ are extremal, we have

$$
\varphi(\alpha(X, Y))=\varphi(g(X, Y) H)=0=\bar{\alpha}(X, Y)
$$

for all $X \in T_{1}^{\top}$ and $Y \in T_{-1}^{\top}$. Therefore $\varphi$ preserves the second fundamental forms. Consider a connection $\bar{\nabla}^{\perp \prime}$ on the normal bundle $\overline{T^{\perp} M}$ defined by $\bar{\nabla}_{X}^{\perp \prime} \xi$ := $\varphi\left(\nabla_{X}^{\perp} \varphi^{-1} \xi\right)$ for $X \in \Gamma(T M)$ and $\xi \in \Gamma\left(\overline{T^{\perp} M}\right)$. By the Codazzi equation (2) for $f$, we see that the connection $\bar{\nabla}^{\perp \prime}$ satisfies $d^{\bar{\nabla}^{\perp \prime}} \alpha=0$. From Lemma 10, it follows that $\bar{\nabla}^{\perp}=\bar{\nabla}^{\perp \prime}$. Therefore $\varphi$ also preserves the normal connections. By the congruence theorem for pseudo-Riemannian submanifolds in the space forms (see [6], for example), we see that there exists an isometry $\Phi$ of $Q_{2}^{4}(c)$ such that $\bar{f}=\Phi \circ f$.

By Theorem 2, we have

Corollary 2. Let $f, \bar{f}: M \rightarrow Q_{2}^{4}(c)$ be super-extremal immersions from a Lorentzian surface $M$ of constant Gaussian curvature $c^{\prime}$. If $c \neq c^{\prime}$, then there exist an isometry $\Phi$ of $Q_{2}^{4}(c)$ such that $\bar{f}=\Phi \circ f$.

An isometric immersion $f:(M, g) \rightarrow(\tilde{M}, \tilde{g})$ is called locally homogeneous if for all point $x$ and $y$ of $M$, there exists a neighborhood $U$ of $x$ and an isometry $\Phi: \tilde{M} \rightarrow \tilde{M}$ such that $\Phi(f(x))=f(y)$ and $\Phi(f(U)) \subset f(M)$. In particular, when $U=M, f$ is said to be homogenous. By Corollary 2, we have 
Corollary 3. Let $f: M \rightarrow Q_{2}^{4}(c)$ be a super-extremal immersion from a Lorentzian surface $M$ of constant Gaussian curvature $c^{\prime}$. If $c \neq c^{\prime}, f$ is locally homogeneous. In particular, if $M=Q_{1}^{2}\left(c^{\prime}\right)$ and $c \neq c^{\prime}$, then $f$ is homogeneous.

For locally homogeneous super-extremal surfaces, a quantization phenomenon of the Gaussian curvature of $M$ holds.

Lemma 11. Let $f: M \rightarrow Q_{2}^{4}(c)$ be a locally homogeneous super-extremal immersion from a Lorentzian surface $M$. We have $K=c$ or $K=c / 3$.

Proof: Take vectors $X \in T_{1}^{\top}, Y \in T_{-1}^{\top}, \xi \in T_{1}^{\perp}$ and $\eta \in T_{-1}^{\perp}$ such that $g(X, Y)=1$ and $g^{\perp}(\xi, \eta)=1$ at each point of $M$. We define

$$
s:=g^{\perp}(\alpha(X, X), \eta) g^{\perp}(\alpha(Y, Y), \xi) .
$$

Note that the function $s$ is independent of the choice of such frames. Since $f$ is locally homogeneous, $s$ is constant. If $s=0$, then we see that $\alpha(X, X)=0$ or $\alpha(Y, Y)=0$. From the Gauss equation (1), we have $K=c$. If $s \neq 0$, then we have $0=\triangle \log |s|=\left(2 K-K^{\perp}\right)$ by Proposition 3.4.1 in [12] (we use the opposite sign convention to the definition of the normal curvature of [12]). By (4), we have $c=3 K$.

By Corollary 3 and Lemma 11, we characterize the immersion of the Veronese type as follows.

Corollary 4. Let $f: M \rightarrow Q_{2}^{4}(c)$ be a super-extremal immersion from a Lorentzian surface $M$ of constant Gaussian curvature $c^{\prime}$. If $c^{\prime} \neq c$, then $c=3 c^{\prime} \neq 0$ and $f$ is congruent to a restriction to an open set of the immersion of the Veronese type given in Example 3.

Remark 1. From Corollary 4, there are no super-extremal immersions of the Lorentzian surfaces of constant Gaussian curvature $c^{\prime}$ into $Q_{2}^{4}(c)$ if $c c^{\prime}<0$.

Remark 2. In Riemannian case, superminimal surfaces in the spaces of constant curvature with flat normal connection are totally geodesic (see Lemma 4.5 in [7], for example). But the corresponding fact does not hold in general. In fact, there exists a non-totally geodesic super-extremal surface in $\mathbb{R}_{2}^{4}$ with flat normal connection. For example, consider the case of $d=0$ in Example 2. By the straightforward calculation, we have

$$
f_{*}\left(\partial_{x}\right)=\left(a^{\prime}, b^{\prime}, a^{\prime}, b^{\prime}\right), \quad f_{*}\left(\partial_{y}\right)=\left(c^{\prime}, 0,-c^{\prime}, 0\right)
$$


and the induced metric satisfies

$$
g\left(\partial_{x}, \partial_{x}\right)=0, \quad g\left(\partial_{y}, \partial_{y}\right)=0, \quad g\left(\partial_{x}, \partial_{y}\right)=-2 a^{\prime} c^{\prime}
$$

Then the Gaussian curvature of the induced metric is flat, and hence $K^{\perp}=0$. Moreover it holds that

$$
\alpha\left(\partial_{x}, \partial_{x}\right)=\left(0, b^{\prime \prime}-\frac{a^{\prime \prime}}{a^{\prime}} b^{\prime}, 0, b^{\prime \prime}-\frac{a^{\prime \prime}}{a^{\prime}} b^{\prime}\right), \quad \alpha\left(\partial_{x}, \partial_{y}\right)=0 \quad \alpha\left(\partial_{y}, \partial_{y}\right)=0
$$

and hence we can find many functions $a, b, c$ such that $M$ is not totally geodesic, for example,

$$
a(x)=x, \quad b(x)=\sin x, \quad c(y)=-\mathrm{e}^{y} .
$$

In Riemannian case, a connected minimal surface of constant Gaussian curvature in $\mathbb{R}^{n}\left(\cong \mathbb{R}_{0}^{n}\right)$ must be flat and an open part of a two-plane on $\mathbb{R}^{n}$ (see [2]). The example as above implies that the corresponding fact does not hold in general. In fact, by Corollary 4, we can see that super-extremal Lorentzian surfaces of constant Gaussian curvature in $\mathbb{R}_{2}^{4}$ must be flat but there are many non-totally geodesic super-extremal flat surfaces. Moreover the immersion given by (6) shows that there exists a non-homogeneous super-extremal flat surface in $\mathbb{R}_{2}^{4}$. So the condition $c \neq c^{\prime}$ in Corollary 3 is needed in general.

\section{Acknowledgements}

The author would like to thank to the organizers of the Thirteenth International Conference "Geometry, Integrability and Quantization" for giving him the opportunity to present his results. This work is partially supported by the Grant-in-Aid for Young Scientists (B) \# 20740046, the Ministry of Education, Culture, Sports, Science and Technology of Japan.

\section{References}

[1] Bryant R., Conformal and Minimal Immersions of Compact Surfaces into Four-Sphere, J. Diff. Geom. 17 (1982) 455-473.

[2] Bryant R., Minimal Surfaces of Constant Curvature in $S^{n}$, Trans. Amer. Math. Soc. 290 (1985) 259-271.

[3] Calabi E., Minimal Immersions of Surfaces in Euclidean Spheres, J. Diff. Geom. 1 (1967) 111-125. 
[4] Friedrich T., On Surfaces in Four-Spaces, Ann. Global Anal. Geom. 2 (1984) 275-287.

[5] Friedrich T., On Superminimal Surfaces, Arch. Math. 33 (1997) 41-56.

[6] Hasegawa K., The Fundamental Theorems for Affine Immersions into Hyperquadrics and its Applications, Monatsh. Math. 131 (2000) 37-48.

[7] Hasegawa K., On Surfaces whose Twistor Lifts are Harmonic Sections, J. Geom. Phys. 57 (2007) 1549-1566.

[8] Hasegawa K., Stability of Twistor Lifts for Surfaces in Four-Dimensional Manifolds as Harmonic Sections, J. Geom. Phys. 59 (2009) 1326-1338.

[9] Hasegawa K., On Surfaces of Low Genus Whose Twistor Lifts are Harmonic Sections, J. Geom. Symmetry Phys. 17 (2010) 35-43.

[10] Hasegawa K., Surfaces in Four-Dimensional Hyperkähler Manifolds Whose Twistor Lifts are Harmonic Sections, Proc. Amer. Math. Soc. 139 (2011) 309317.

[11] Hasegawa K. and Miura K., Lorents Surfaces in Pseudo-Riemannian Space Forms of Neutral Sugnature with Horizontal Reflector Lifts (in preparation).

[12] Jensen G. and Rigoli M., Neutral Surfaces in Neutral Four-Spaces, Matematiche (Catania) 45 (1990) 407-443.

[13] Magid M., Isometric Immersions of Lorentzian Space with Parallel Second Fundamental Forms, Tsukuba J. Math. 8 (1984) 31-54.

[14] Miura K., Construction of Harmonic Maps Between Semi-Riemannian Spheres, Tsukuba J. Math. 31 (2007) 397-409.

[15] Miura K., Helical Geodesic Immersions of Semi-Riemannian Manifolds, Kodai Math. J. 30 (2007) 322-343.

[16] Nomizu K., Uniqueness of the Normal Connections and Congruence of Isometric Immersions, Tôhoku Math. J. 28 (1976) 613-617.

[17] O’Neill B., Semi-Riemannian Geometry, Pure and Applied Mathematics, 103 Academic Press, New York 1983.

Kazuyuki Hasegawa

Faculty of Teacher Education

Institute of Human and Social Sciences

Kanazawa University, Kakuma-machi

Kanazawa, Ishikawa, 920-1192

JAPAN

E-mail address: kazuhase@staff.kanazawa-u.ac.jp 